\title{
Up-regulation of prohibitin 1 is involved in the proliferation and migration of liver cancer cells
}

\author{
XU ZhiBin ${ }^{1 \dagger}$, WU JinHong ${ }^{2 \dagger} \&$ ZHA XiLiang ${ }^{3 *}$ \\ ${ }^{1}$ Institute of Biomedical Sciences, Fudan University, Shanghai 200032, China; \\ ${ }^{2}$ School of Agriculture and Biology, Bor Luh Food Safety Center, Shanghai Jiaotong University, Shanghai 200030, China; \\ ${ }^{3}$ Key Laboratory of Glycoconjugate, Ministry of Health, Department of Biochemistry and Molecular Biology, \\ Shanghai Medical College, Fudan University, Shanghai 200032, China
}

Received October 4, 2010; accepted November 11, 2010

\begin{abstract}
Although prohibitin 1 (PHB) is known to be associated with tumors, there are few studies regarding the role of PHB in hepatocellular carcinoma. An earlier glycoproteomics study of ours showed that PHB is over-expressed in MHCC 97-H cells, a highly metastatic liver cancer cell line, and can be captured by wheat germ agglutinin. In the present study, western blotting and reverse transcription-PCR experiments showed an approximately 2-fold up-regulation in the expression of PHB in MHCC97-H cells. However, PHB was not significantly up-regulated in MHCC97-L cells, a low-metastatic liver cancer cell line. When PHB was over-expressed in MHCC97-L cells, cell proliferation was inhibited by 35\% and migration increased about 2-fold. The results of this study show that PHB is up-regulated in MHCC97-H cells and is associated with both proliferation and migration of liver cancer cells.
\end{abstract}

glycosylation, lectin, metastasis, prohibitin, up-regulation

Citation: $\quad \mathrm{Xu} \mathrm{Z} \mathrm{B,} \mathrm{Wu} \mathrm{J} \mathrm{H,} \mathrm{Zha} \mathrm{X} \mathrm{L.} \mathrm{Up-regulation} \mathrm{of} \mathrm{prohibitin} 1$ is involved in the proliferation and migration of liver cancer cells. Sci China Life Sci, 2011, 54: 121-127, doi: 10.1007/s11427-010-4130-1

Prohibitin 1 (PHB) is a highly conserved protein in eukaryotic cells that is present in multiple cellular compartments. It belongs to the prohibitin family, which comprises PHB and prohibitin 2. PHB has a molecular mass of about $32 \mathrm{kD}$ and prohibitin 2 has a molecular mass of about $37 \mathrm{kD}$ [1]. Together, these proteins can form a high molecular weight complex. These complexes have been identified in mitochondria [2], the plasma membrane [3] and the nucleus [4]. There is some controversy concerning the expression levels of PHB by different tumors and cell lines. For example, decreased expression of PHB was reported in human stomach adenocarcinoma tissue compared with the surrounding normal tissues [5]. However, a number of

${ }^{\dagger}$ Contributed equally to this work

*Corresponding author (email: xlzha@shmu.edu.cn) studies report high-level expression of PHB in other tumor tissues, such as lung cancer, prostate carcinoma [6], and rat bladder carcinomas [7]. Studies have also shown that PHB levels are higher in transformed cells and tumors than in their normal counterparts [7-9]. Therefore, it is possible that PHB expression is associated with cancer.

In previous studies, we used wheat germ agglutinin (WGA) affinity chromatography to capture the N-linked glycoproteins expressed by Chang liver cells (immortalized human non-tumor liver cells) and MHCC97-H cells (a highly metastatic liver cancer cell line). Using two-dimensional electrophoresis (2-DE) and mass spectrometry (MS), we separated and identified five markedly up-regulated glycoproteins from MHCC97-H cells (published in the journal of Proteomics) [10]. Of these, we focused on a new glycoprotein, PHB. 
The aim of the present study was to confirm that PHB was over-expressed in MHCC97-H cells and to study the role of $\mathrm{PHB}$ in the proliferation and migration of liver cancer cells.

\section{Materials and methods}

\subsection{Materials}

RPMI 1640 and DMEM were purchased from Gibco (USA). Chang liver cells, MHCC97-H cells, and MHCC97-L cells were obtained from the Liver Cancer Institute, Zhongshan Hospital, Fudan University. An unconjugated mouse monoclonal anti-prohibitin 1 antibody was purchased from Novus Biologicals (USA) (Clone II-14-10, ab1836). Lipofectin 2000 was purchased from Invitrogen (USA). Trizol reagent for total RNA extraction was purchased from Watson (Shanghai). RT-PCR kits, and Xba I and BamH I endonucleases were obtained from Takara (Japan). The ECL detection reagent was purchased from Amersham Biosciences (Sweden). All other chemicals were analytical grade products obtained commercially.

\subsection{Cell culture}

Chang liver cells were cultured at $37^{\circ} \mathrm{C}$ in $5 \% \mathrm{CO}_{2}$ in RPMI 1640 supplemented with $10 \%$ newborn calf serum. MHCC97-H and MHCC97-L cells were established from the parent cell line, MHCC97. MHCC97-H cells, MHCC97-L cells, and other cells were cultured at $37^{\circ} \mathrm{C}$ in $5 \% \mathrm{CO}_{2}$ in DMEM supplemented with $10 \%$ fetal bovine serum.

\subsection{WGA affinity chromatography, 2-DE and MS}

WGA affinity chromatography, 2-DE and MS analysis were performed as previously described [10]. Briefly, agarosebound WGA (Vector Laboratories, Al-1023) was prepared and used to capture glycoproteins from the soluble fractions of Chang liver and MHCC97-H lysates. The eluted fractions were dialyzed with Milli Q water (Millipore, USA) at $4^{\circ} \mathrm{C}$ and freeze-dried. 2-DE was performed as previously described [10] using the IPGphor IEF System and a Hoefer SE 600 (Amersham Biosciences, Sweden). Glycoproteins $(250 \mu \mathrm{g})$ were assessed by isoelectric focusing (IEF) using $13 \mathrm{~cm} \mathrm{pH}$ 3-10 NL IPG strips (Amersham Biosciences). The separated proteins were visualized using silver diamine-staining as described in the Amersham 2-DE protocol. For MS detection, $1 \mathrm{mg}$ of glycoproteins was separated as described above and the proteins were stained with Coomassie Brilliant Blue (CBB) R250. MALDI-TOF MS and tandem TOF/TOF MS were performed using a 4700 MALDI-TOF/TOF Proteomics Analyzer (Applied Biosystems, USA). The measured peptide mass profiles were compared with the theoretical peptide masses using the MASCOT (www.matrixscience.com) search engine and
IPI-HUMAN and NCBI databases for protein identification. The 2-DE gels were scanned using an ImageScanner ${ }^{\mathrm{TM}}$ (Amersham Biosciences) and data were analyzed using ImageMaster $^{\mathrm{TM}}$ 2-D Platinum software (v5.0, Amersham Biosciences).

\subsection{Western blot analysis}

Cells were washed three times in PBS at $4{ }^{\circ} \mathrm{C}$ and lysed in lysis buffer $\left(50 \mathrm{mmol} \mathrm{L}^{-1}\right.$ Tris- $\mathrm{HCl}, \mathrm{pH} 6.8,2 \% \mathrm{SDS}, 10 \%$ glycerol, $1 \mathrm{mmol} \mathrm{L}{ }^{-1}$ PMSF, $1 \mu \mathrm{g} \mathrm{mL}^{-1}$ leupeptin, and $1 \mu \mathrm{g}$ $\mathrm{mL}^{-1}$ aprotinin). Lysates were clarified by centrifugation at $15000 \times \mathrm{g}$ for $10 \mathrm{~min}$ at $4^{\circ} \mathrm{C}$. The protein concentration was determined using a modified Lowry assay $[11,12]$. Proteins $(60 \mu \mathrm{g})$ were run on a $10 \%$ SDS polyacrylamide gel and electrophoretically transferred onto a nitrocellulose membrane. The membrane was blocked for $4 \mathrm{~h}$ in PBS containing $0.05 \%$ Tween 20 (PBST) containing 5\% nonfat milk and incubated overnight with a mouse anti-prohibitin primary antibody (ab1836, diluted 1:1000) and a mouse antiglyceraldehyde-3-phosphate dehydrogenase (GADPH, diluted 1:4000) antibody. After washing, the nitrocellulose membrane was incubated with horseradish peroxidase (HRP)-conjugated anti-mouse $\operatorname{IgG}(1: 3000)$ for $4 \mathrm{~h}$. After washing the membrane three times with PBST, proteins were visualized using enhanced chemiluminescence (ECL) detection reagents according to the manufacturer's instructions. The results were analyzed using TotalLab software.

\subsection{Reverse transcription (RT)-PCR, plasmid con- struction and transfections}

Total RNA samples were prepared using Trizol reagent. RT-PCR was carried out according to the manufacturer's instructions. Briefly, $4 \mu \mathrm{g}$ of RNA was used for the RT reaction and $2 \mu \mathrm{L}$ of the cDNA product was used in the PCR mix along with a forward primer, 5'-GCGGATCCATGGCTGCCAAAGTGTTTGAGT-3' and a reverse primer, 5'GCTCTAGACTACTGGGGCAGCTGGAGGAGC-3'. Primers for $\beta$-actin were used as an internal control. Samples were amplified for 30 cycles of $95^{\circ} \mathrm{C}$ for $30 \mathrm{~s}, 65^{\circ} \mathrm{C}$ for $30 \mathrm{~s}$, and $72^{\circ} \mathrm{C}$ for $3 \mathrm{~min}$. The PCR products were analyzed on $1 \%$ agarose gels. PHB cDNA was amplified using PHBspecific primers containing Xba I and BamH I restriction sites. The purified 819-bp fragment and the pcDNA3.0 vector were cut with $X b a$ I and BamH I and ligated to yield the recombinant PHB expression plasmid, pcDNA3.0-PHB. Purified plasmid DNA was transfected into MHCC97-L cells using Lipofectin 2000 according to the manufacturer's instructions.

\subsection{MTT assay}

A colorimetric assay using the tetrazolium salt 3-(4,5Dimethylthiazol-2-yl)-2,5-diphenyltetrazolium bromide (MTT) 
was used to determine the effects of PHB on MHCC97-L cell proliferation. The MTT assay was performed as previously described [13]. Briefly, after transfection with pcDNA3.0 and pcDNA3.0-PHB for $12 \mathrm{~h}$, an equal number of cells were seeded into a 96-well plate at a density of $5 \times 10^{3}$ cells per well. After $24 \mathrm{~h}, 48 \mathrm{~h}$ and $72 \mathrm{~h}$ of incubation, $20 \mu \mathrm{L}$ of MTT (stock solution $5 \mathrm{mg} \mathrm{mL}^{-1}$ ) was added to each well. After a further $4 \mathrm{~h}$, DMSO $(150 \mu \mathrm{L})$ was added to each well to dissolve the crystals. The optical density $(A)$ of each well was read using an enzyme-linked immunosorbent assay reader (Bio-Tek, USA) at $490 \mathrm{~nm}$. All experiments were performed in triplicate. The cell inhibitory ratio was calculated using the following formula: Inhibitory ratio $=\left(1-A_{490}\right.$ Treated group $/ A_{490}$ Control group $) \times$ $100 \%$.

\subsection{Transwell migration assay}

MHCC97-L cells were transfected with plasmids in serum-free medium. After $24 \mathrm{~h}$, a transwell assay was used to monitor in vitro cell migration as previously described [12]. Briefly, $1 \times 10^{4}$ tumor cells in $0.5 \%$ BSA-DMEM were placed in the upper chamber of the transwell unit and $0.5 \%$ BSA-DMEM containing $0.5 \%$ FBS was placed in the lower chamber. After a $12-\mathrm{h}$ incubation at $37^{\circ} \mathrm{C}$ in $5 \% \mathrm{CO}_{2}$ the cells were fixed in methanol and the upper side of the filter removed and gently wiped with a cotton swap. Cell migration was determined by counting the cells that migrated to the lower side of the polycarbonate filters using a standard crystal violet staining technique (the cell numbers in eight random areas were counted under a light microscope at $\times 10$ magnification). Each assay was repeated three times.

\subsection{Statistical analysis}

All data were analyzed using one-way analysis of variance (ANOVA) and SPSS 12.0 for Windows ${ }^{\circledR}$ software (SPSS Inc., USA). A $P$-value of $>0.05$ was considered significant. Post-hoc analysis was done using Tukey's Honestly Significant difference (HSD) test. All curves were produced using Microsoft ${ }^{\circledR}$ Office Excel (Microsoft Corporation, USA) and the $Y$-axis error bars represent $P<0.05$.

\section{Results}

\subsection{Prohibitin is up-regulated in MHCC97-H cells}

Figure 1 shows enlarged 2-DE images of the PHB proteins from Chang liver and MHCC97-H cells captured by WGA. The spots were analyzed using ImageMaster ${ }^{\mathrm{TM}}$ 2-D Platinum software and the results showed that PHB was upregulated (2.64 \pm 0.12 )-fold in MHCC97-H cells compared with Chang liver cells $(P<0.05)$. The prohibitin spots were cut from CBB R250 stained gels for identification using
MALDI-TOF MS. Figure 2 shows the identification prohibitin by PMF and MS/MS and Figure 2A shows the mass fingerprints. The product ion spectra of the tryptic peptides T-(1-10) FDAGELITQR at $\mathrm{m} / z, 1149.6158$ and T-(1-12) ILFRPVASQLPR at $m / z, 1396.8728$ are shown in Figure 2B and $\mathrm{C}$, respectively. MASCOT searching indicated that 10 and 12 peptides matched the peptides identified from the spots. The sequence coverage was $79 \%$. The MASCOT score was as high as $422(P<0.05)$, implying significant confidence levels for the identification. Both the MS and MS/MS analyses showed that the best match for the protein spot was prohibitin [10].

We confirmed that PHB was up-regulated in MHCC97-H cells using RT-PCR and western blotting. RT-PCR showed that PHB mRNA levels increased $(1.80 \pm 0.12)$-fold in MHCC97-H cells compared with Chang liver cells $(P<0.05$; Figure $3 \mathrm{~A})$. After lysis of the cells, the extracts were analyzed by western blotting. The results showed that PHB was

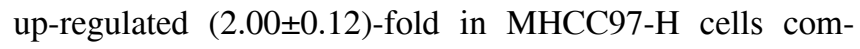
pared with Chang liver cells $(P<0.05)$ (Figure $3 \mathrm{~B})$. These results showed that $\mathrm{PHB}$ was up-regulated at both the transcriptional and translational levels.

\subsection{PHB is associated with the migration of liver can- cer cells}

MHCC97-H and MHCC97-L cells were established from the parent cell line, MHCC97. The two daughter cell lines had the same genetic background, but different metastatic

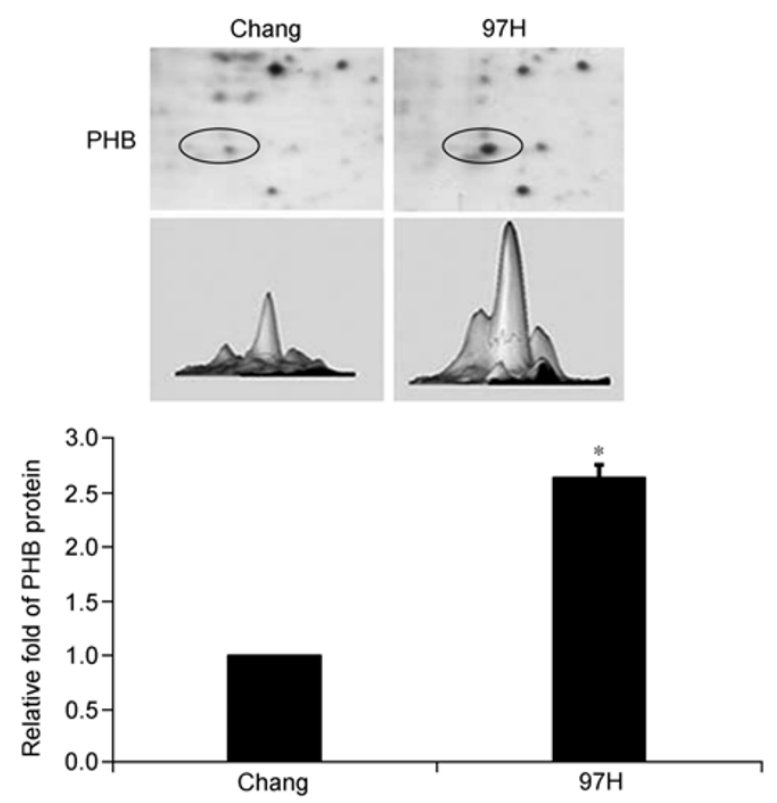

Figure 1 Comparative analysis of PHB expression in 2-DE gels. The up-regulated spots are shown in the enlarged images of the 2-DE gels. Upper panel, PHB protein spots; lower panel, spot analysis using ImageMaster $^{\mathrm{TM}}$ 2-D Platinum software. The relative abundance is shown as a 3-dimensional simulation. The $Y$ axis indicates the over-expression of PHB protein relative to the control (Chang liver cells). Data are expressed as the mean $\pm \mathrm{SD}(n=3)$. * $P<0.05$ (compared with Chang liver cells). 

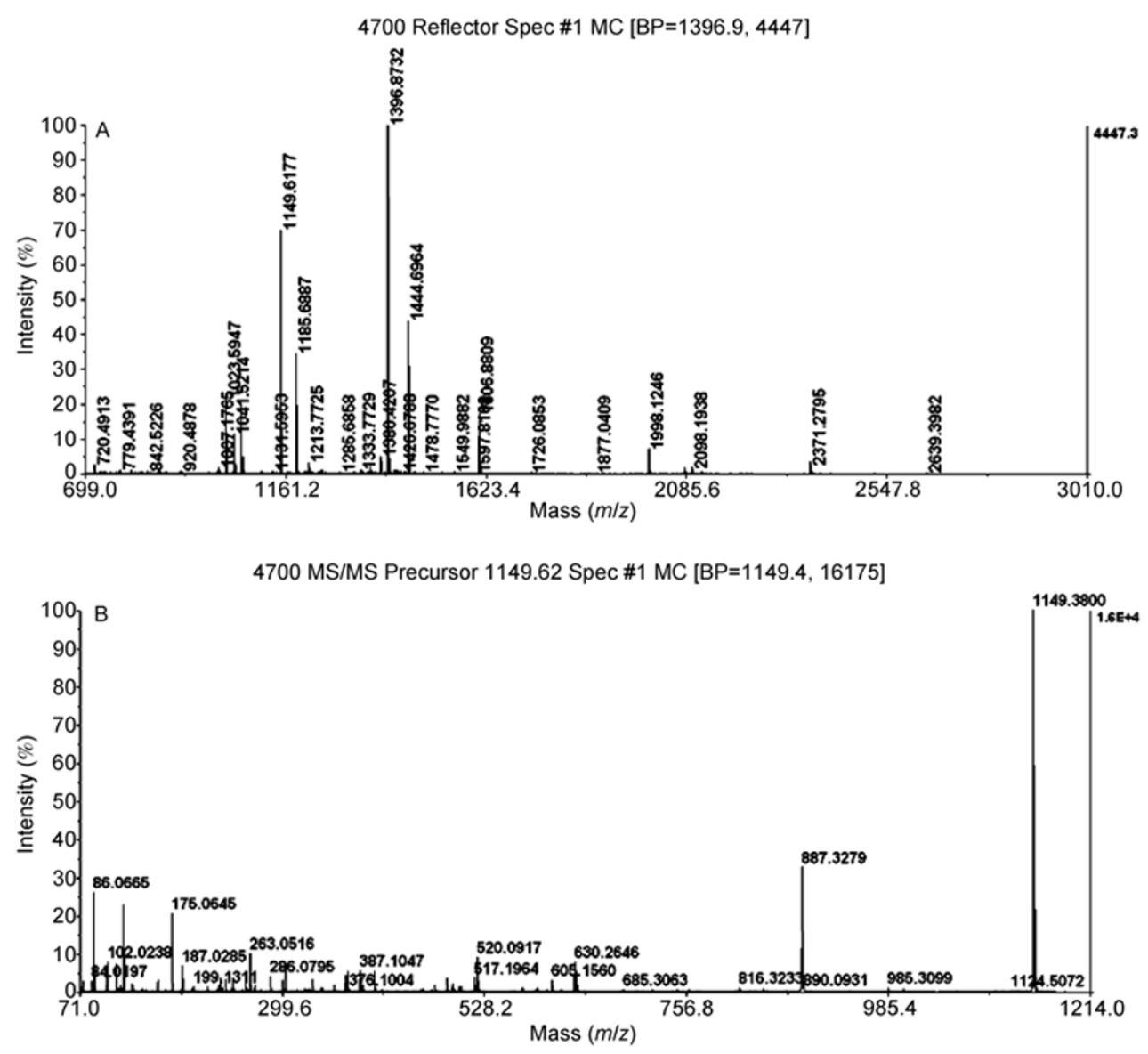

4700 MS/MS Precursor 1395.87 Spec \#1 MC [BP=1396.6, 27972]

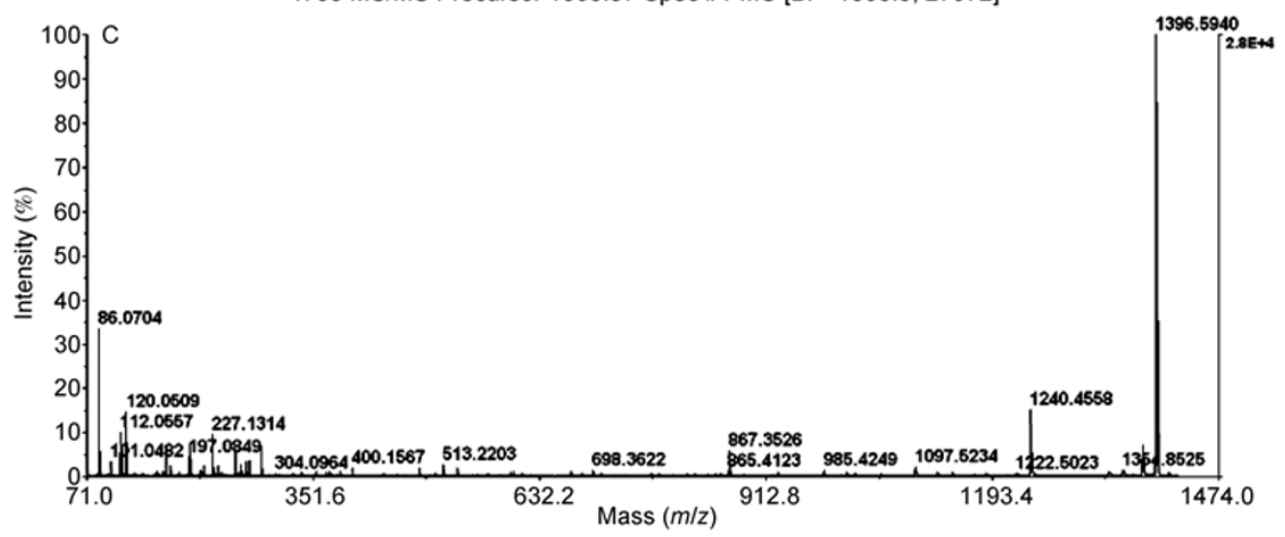

Figure 2 Identification of prohibitin protein by PMF and MS/MS. A, MALDI peptide mass fingerprint of prohibitin. B, The product ion spectra of the tryptic peptide T-(1-10) FDAGELITQR at $m / z, 1149.6158$ from prohibitin. The ion score was 91 and the sequence coverage reached $79 \%$. C, The product ion spectra of the tryptic peptide T-(1-12) ILFRPVASQLPR at $\mathrm{m} / \mathrm{z} 1396.8728$ from prohibitin. The ion score was 59.

potential. Spontaneous pulmonary metastasis occurred in $100 \%$ and $40 \%$ of recipient nude mice after orthotopic inoculation of MHCC97-H and MHCC97-L cells, respectively $[14,15]$. Therefore, we wondered whether PHB was over-expressed in MHCC97-L cells. The results showed that the expression level of PHB in MHCC97-L cells was not significantly higher than that in Chang liver cells (Figure 3).

However, we did notice different expression levels of PHB between MHCC97-L and MHCC97-H cells. Figure 3B shows that the expression level of PHB in MHCC97-H cells was $1.50 \pm 0.04$ times higher than that in MHCC97-L cells $(P<0.05)$. This suggested that up-regulation of PHB may be involved in the metastasis of liver cancer cells.

\subsection{The influence of PHB over-expression on the pro- liferation and migration of MHCC97-L cells}

Compared with MHCC7-H cells, the expression level of PHB in MHCC97-L cells was low. Therefore, we used 

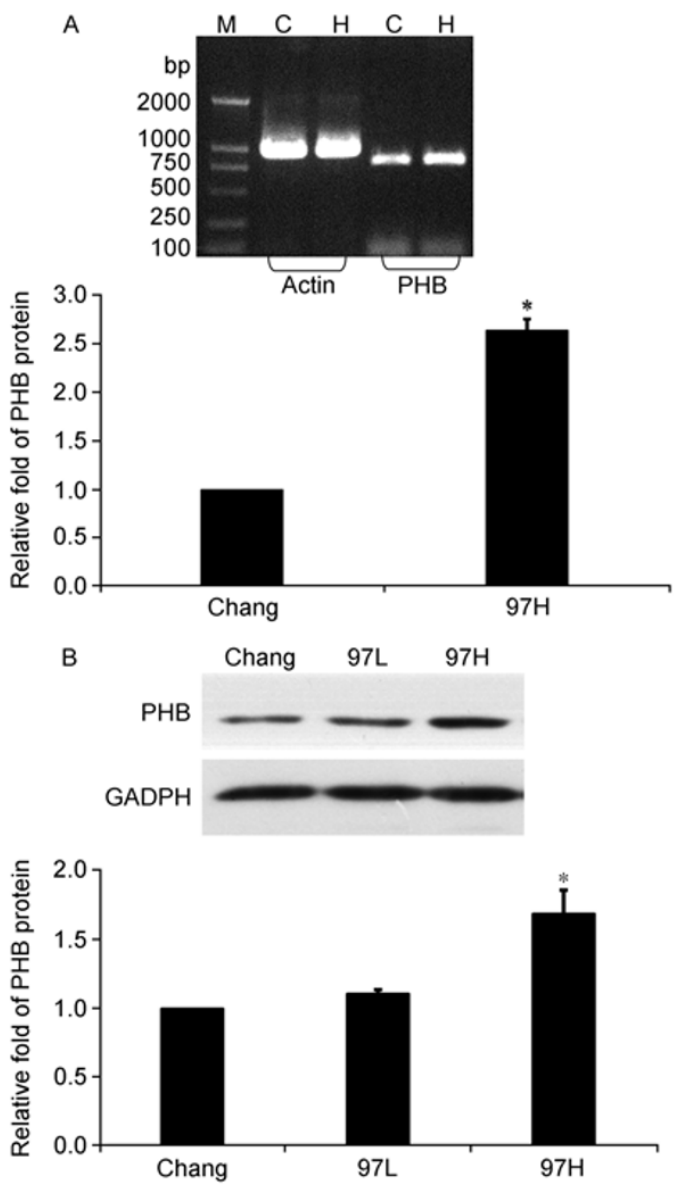

Figure 3 Comparative analysis of PHB mRNA and protein levels in Chang liver cells and MHCC97-H cells. A, Semi-quantitative RT-PCR of PHB mRNA from Chang liver and MHCC97-H cells. Lane 1, marker; lanes 2 and 3, actin; lanes 4 and 5, PHB; M, marker; C, Chang liver cells; $\mathrm{H}$, MHCC97H cells. The $Y$ axis indicates the over-expression of PHB mRNA relative to the control (Chang liver cells). Data are expressed as the mean $\pm \mathrm{SD}(n=3) ; *, P<0.05$ (compared with Chang liver cells). B, Western blot of PHB from Chang liver, MHCC97-L and MHCC97-H cells. Upper panel, PHB protein; lower panel, GADPH. The $Y$ axis indicates the over-expression of the PHB protein relative to the control (Chang liver cells). Data are expressed as the mean $\pm \mathrm{SD}(n=3) ; * P<0.05$ (compared with Chang liver cells).

MHCC97-L cells to study the influence of PHB on the proliferation and migration of liver cancer cells. First, we constructed the pcDNA3.0-PHB plasmid (Figure 4A). After transfection of the plasmid into MHCC97-L cells, we found that PHB was up-regulated $(1.60 \pm 0.10)$-fold $(P<0.05)$ (Figure 4B). As it was reported that over-expression of PHB could inhibit the proliferation of breast epithelial cells [16], we used an MTT assay to study the influence of PHB over-expression on MHCC97-L cell proliferation. The results showed a $35 \%$ inhibition of cell proliferation that lasted up to $72 \mathrm{~h}$ after the transfection (Figure 5). This suggested that PHB also played a role in inhibiting MHCC97-L cell proliferation.

There are few reports regarding the association of PHB with the migration of hepatocellular carcinoma cells. The
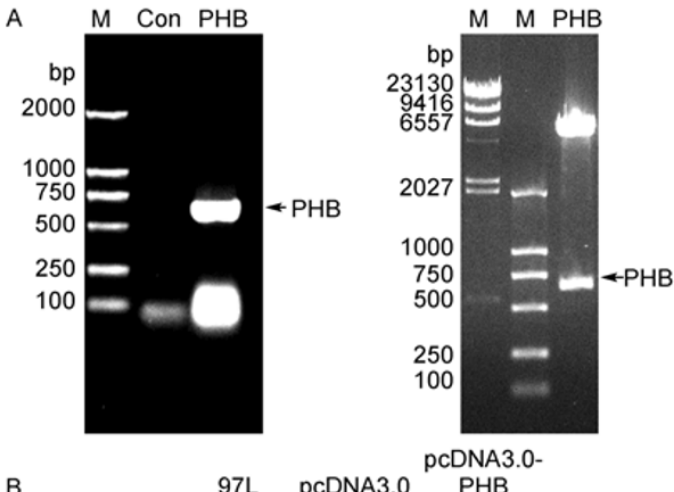

B
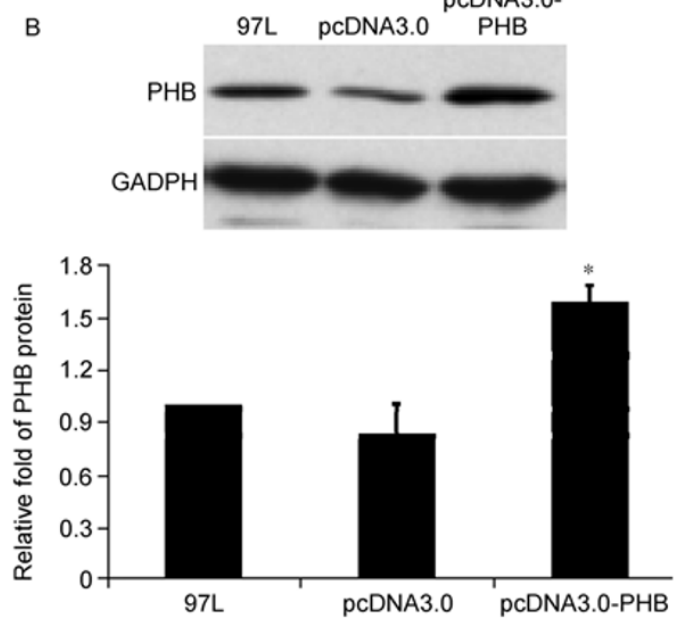

Figure 4 Construction of the PHB over-expression plasmid. A, Left, PHB PCR product amplified from the cDNA of Chang lover cells; Right, PHB cDNA after cutting pcDNA 3.0-PHB with Xba I and BamH I. M, marker; Con, PCR product from control. B, PHB over-expression in transfected MHCC97-L cells was examined by Western blotting. The $Y$ axis indicates the level of the up-regulated PHB protein relative to the control (MHCC97-L cells). Data are expressed as the mean \pm SD $(n=3) ; *, P<0.05$ (compared with MHCC97-L cells).

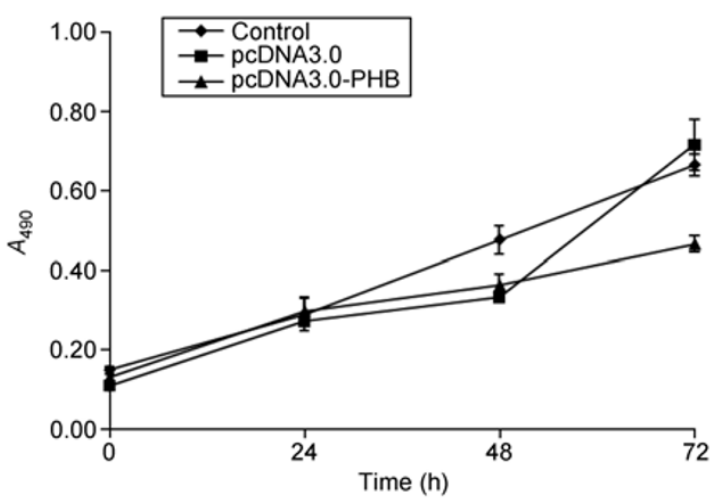

Figure 5 Over-expression of PHB inhibited the proliferation of MHCC97-L cells. MHCC97L cells were cultured in 96-well plates after transfection with pcDNA 3.0-PHB for $24 \mathrm{~h}$. Proliferation assessed using an MTT assay.

metastatic efficiency of tumor cells is evaluated using cell migration assays. Therefore, we assessed the migration of MHCC97-L cells using transwell migration assays. The 

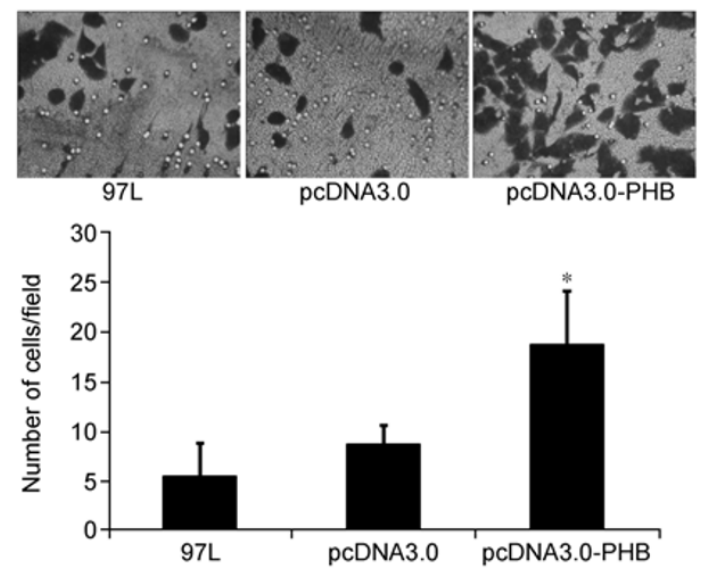

Figure 6 Increased MHCC97-L cell migration induced by over-expression of PHB. The $Y$ axis indicates the number of cells counted within each field ( 8 fields for each group). Data are expressed as the mean \pm SD. *, $P<0.05$ (compared with the MHCC97-L group)

micrographs of a typical transwell experiment (Figure 6) indicated that the migration of pcDNA3.0-PHB-transfected cells was 2.10 \pm 0.10 times higher than that of the pcDNA3.0transfected cells $(P<0.05)$. This suggested that up-regulation of PHB promoted the migration of MHCC97-L cells.

\section{Discussion}

The results of this study showed that PHB was over-expressed in liver cancer cells and inhibited the proliferation, and promoted the migration, of these cells. This raises several questions regarding the over-expression of PHB in tumor cells. PHB was believed to be a mitochondrial chaperone indirectly related to the aging process and carcinogenesis through its effects on the stability of newly encoded mitochondrial proteins and the subsequent assembly of respiratory chain complexes. Given that PHB levels appear to decrease during cellular senescence, it is conceivable that over-expression of PHB might extend the life span of tumor cells, which could be a critical step in the establishment of tumorigenic clones during the early stages of carcinogenesis [17]. Nijtmans et al. [2] provided an alternative explanation for the association between PHB over-expression and proliferation. They suggested that it was because of the presence of regulatory elements within the PHB promoter that bind to the Myc oncoprotein. Up-regulated Myc expression, which is common in proliferating cells, induces the expression of PHB.

Tumor cell migration is a major cause of mortality in cancer patients. Altered expression of cellular adhesion molecules is critical for the migration of primary tumors to the liver. Loss of E-cadherin and increased expression of CD44 variant 6 are associated with hepatic migration and a poor prognosis for primary colorectal liver metastases [18]. In a study of cervical adenocarcinomas, high PHB expres- sion was found in 51 of $69(73.9 \%)$ invasive adenocarcinoma cases and in 11 of $16(68.7 \%)$ adenocarcinoma in situ lesions. PHB over-expression was significantly higher in invasive adenocarcinoma and adenocarcinoma in situ tissues than in the adjacent non-neoplastic glandular epithelium [17]. Rajalingam et al. [19] identified an unexpected role for PHB in the activation of the Ras-Raf signaling pathway and in the modulation of epithelial cell adhesion and migration. In our experiments, we found that PHB was up-regulated in MHCC97-H cells with a high migration potential, and that over-expression of PHB induced the migration of these cells. This indicates that over-expression of PHB might be specifically associated with the migration of liver cancer cells. Knockdown of PHB using RNAi would confirm the effect of PHB on the proliferation and migration of MHCC97-H cells. Unfortunately, it was not possible to transfect MHCC97-H cells with PHB RNAi in this study.

To the best of our knowledge, this is the first report detailing the capture and identification of PHB from cancer cells using WGA. WGA recognizes $\mathrm{N}$-acetyl-glucosamine (GlcNAc) and, also, has affinity for sialic acid (it has affinity for complex N-type tri and tetraantennary-oligosaccharides). In this study, WGA captured more PHB from MHCC97H cells than from Chang liver cells. This indicates that PHB was either over-expressed in MHCC97-H cells, or that it contains more complex N-type tri- and tetraantennary-oligosaccharides in these cells. Although our data showed that PHB was up-regulated in liver cancer cells, it is still possible that this resulted from differences in the glycan content and structure of PHB between Chang liver cells and MHCC97-cells. Studies looking at the function and structure of the glycans within PHB are ongoing.

Although knowledge about the function of PHB in cancer is limited, our results clearly show that PHB is up-regulated in MHCC97-H cells and plays an important role in the proliferation and migration of liver cancer cells.

We wish to thank the Liver Cancer Institute of Fudan University for providing the cell lines. This work was supported by the Shanghai Leading Academic Discipline Project (Grant No. B110), grants from CNHLPP (Grant No. 2005-BAC11A11), and the Scientific Research Foundation for Young Talents of Shanghai Jiaotong University (Grant No. AB150200).

1 Mishra S, Murphy L C, Nyomba B L, et al. Prohibitin: a potential target for new therapeutics. Trends Mol Med, 2005, 11: 192-197

2 Nijtmans L G, Artal S M, Grivell L A, et al. The mitochondrial PHB complex: roles in mitochondrial respiratory complex assembly, ageing and degenerative disease. Cell Mol Life Sci, 2002, 59: 143-155

3 Sharma A, Qadri A. Vi polysaccharide of Salmonella typhi targets the prohibitin family of molecules in intestinal epithelial cells and suppresses early inflammatory responses. Proc Natl Acad Sci USA, 2004, 101: 17492-17497

4 Fusaro G, Dasgupta P, Rastogi S, et al. Prohibitin induces the transcriptional activity of p53 and is exported from the nucleus upon apoptotic signaling. J Biol Chem, 2003, 278: 47853-47861

5 Jang J S, Cho H Y, Lee Y J, et al. The differential proteome profile of stomach cancer: identification of the biomarker candidates. Oncol Res, 2004, 14: 491-499 
6 Mengwasser J, Piau A, Schlag P, et al. Differential immunization identifies PHB1/PHB2 as blood-borne tumor antigens. Oncogene, 2004, 23: 7430-7435

7 Asamoto M, Cohen S M. Prohibitin gene is overexpressed but not mutated in rat bladder carcinomas and cell lines. Cancer Lett, 1994, 83: 201-207

8 Byrjalsen I, Mose Larsen P, Fey S J, et al. Two-dimensional gel analysis of human endometrial proteins: characterization of proteins with increased expression in hyperplasia and adenocarcinoma. Mol Hum Reprod, 1999, 5: 748-756

9 Seow T K, Ong S E, Liang R C, et al. Two-dimensional electrophoresis map of the human hepatocellular carcinoma cell line, HCC-M, and identification of the separated proteins by mass spectrometry. Electrophoresis, 2000, 21: 1787-1813

$10 \mathrm{Xu} \mathrm{Z}$, Zhou X, Lu H, et al. Comparative glycoproteomics based on lectins affinity capture of N-linked glycoproteins from human Chang liver cells and MHCC97-H cells. Proteomics, 2007, 7: 2358-2370

11 Lowry O H, Rosebrough N J, Farr A L, et al. Protein measurement with the Folin phenol reagent. J Biol Chem, 1951, 193: 265-275

$12 \mathrm{Wu} \mathrm{N}$, Zhang W, Yang Y, et al. Profilin 1 obtained by proteomic analysis in all-trans retinoic acid-treated hepatocarcinoma cell lines is involved in inhibition of cell proliferation and migration. Proteomics,
2006, 6: 6095-6106

13 Mosmann T. Rapid colorimetric assay for cellular growth and survival: application to proliferation and cytotoxicity assays. J Immunol Methods, 1983, 65: 55-63

14 Li Y, Tang Z Y, Ye S L, et al. Establishment of cell clones with different metastatic potential from the metastatic hepatocellular carcinoma cell line MHCC97. World J Gastroenterol, 2001, 7: 630-636

15 Tang Z Y, Sun F X, Tian J, et al. Metastatic human hepatocellular carcinoma models in nude mice and cell line with metastatic potential. World J Gastroenterol, 2001, 7: 597-601

16 Peng X, Mehta R, Wang S, et al. Prohibitin is a novel target gene of vitamin $\mathrm{D}$ involved in its antiproliferative action in breast cancer cells. Cancer Res, 2006, 66: 7361-7369

17 Williams K, Chubb C, Huberman E, et al. Analysis of differential protein expression in normal and neoplastic human breast epithelial cell lines. Electrophoresis, 1998, 19: 333-343

18 Neal C P, Garcea G, Doucas H, et al. Molecular prognostic markers in resectable colorectal liver metastases: a systematic review. Eur J Cancer, 2006, 42: 1728-1743

19 Rajalingam K, Wunder C, Brinkmann V, et al. Prohibitin is required for Ras-induced Raf-MEK-ERK activation and epithelial cell migration. Nat Cell Biol, 2005, 7: 837-843

Open Access This article is distributed under the terms of the Creative Commons Attribution License which permits any use, distribution, and reproduction in any medium, provided the original author(s) and source are credited. 